\section{Relationship between early and late stages of information processing: an event-related potential study}

\author{
Claudio Portella,1,2 \\ Sergio Machado, 3,4,5,6 \\ Oscar Arias-Carrión, 7 \\ Alexander T. Sack, 8 \\ Julio Guilherme Silva,9 Marco Orsini,10,11 \\ Marco Antonio Araujo Leite, 2 \\ Adriana Cardoso Silva, 3 \\ Antonio E. Nardi,3 Mauricio Cagy,12 \\ Roberto Piedade, ${ }^{1}$ Pedro Ribeiro' \\ 1Brain Mapping and Sensory Motor \\ Integration, Institute of Psychiatry of \\ Federal University of Rio de Janeiro, \\ Brazil; 2Faculty of Physical Therapy, UNI- \\ ABEU, Rio de Janeiro, Brazil; ${ }^{3}$ Panic and \\ Respiration, Institute of Psychiatry of \\ Federal University of Rio de Janeiro, \\ National Institute for Translational \\ Medicine (INCT-TM), Brazil; ${ }^{4}$ Quiropraxia \\ Program, Central University, Santiago, \\ Chile; 5 Physical Activity Neuroscience, \\ Physical Activity Sciences Postgraduate \\ Program - Salgado de Oliveira University, \\ Niterói, Brazil; Institute of Phylosophy, \\ Federal University of Uberlândia \\ (IFILO/UFU), Brazil; 7Movement \\ Disorders and Transcraneal Magnetic \\ Stimulation Unit, Hospital General Dr. \\ Manuel Gea González, México DF, México; \\ 8Department of Cognitive Neuroscience, \\ Faculty of Psychology and Neuroscience, \\ Maastricht University, The Netherlands; \\ 9Physical Therapy Department, Federal \\ University of Rio de Janeiro, Brazil; \\ 10Neurology Department, Antonio Pedro \\ University Hospital, Federal Fluminense \\ University, Rio de Janeiro, Brazil; \\ 11Master Program of Science \\ Rehabilitation UNISUAM, Rio de Janeiro, \\ Brazil; 12Division of Epidemiology and \\ Biostatistic, Institute of Health \\ Community, Federal Fluminense \\ University, Rio de Janeiro, Brazil
}

\section{Abstract}

The brain is capable of elaborating and executing different stages of information processing. However, exactly how these stages are processed in the brain remains largely unknown. This study aimed to analyze the possible correlation between early and late stages of information processing by assessing the latency to, and amplitude of, early and late event-related potential (ERP) components, including P200, N200, premotor potential (PMP) and P300, in healthy participants in the context of a visual oddball paradigm. We found a moderate positive correlation among the latency of P200 (electrode 02), N200 (electrode 02), PMP (electrode C3), P300 (electrode PZ) and the reaction time (RT). In addition, moderate negative correlation between the amplitude of P200 and the latencies of N200 (electrode 02), PMP (electrode C3), P300 (electrode PZ) was found. Therefore, we propose that if the secondary processing of visual input (P200 latency) occurs faster, the following will also happen sooner: discrimination and classification process of this input (N200 latency), motor response processing (PMP latency), reorganization of attention and working memory update (P300 latency), and RT. N200, PMP, and P300 latencies are also anticipated when higher activation level of occipital areas involved in the secondary processing of visual input rise (P200 amplitude).

\section{Introduction}

Recent studies highlighted the importance of central organization of neural processes involved in what has been called the different stages of information processing. ${ }^{1-11}$ The brain is capable of processing and executing these different stages in a seemingly simple and efficient manner, i.e. the brain detects, identifies and discriminates different stimuli, and subsequently selects and activates appropriate responses in a matter of hundreds of a millisecond.1,2 However, exactly how these information-processing stages are processed in the brain remains largely unknown. Several researchers have argued that these processes occur serially, with the completion of one stage being a requirement for the beginning of the next. ${ }^{8}$ In line with this notion, one would expect that impairments in initial stages of sensory information processing would affect the subsequent phase(s); perhaps also affecting cognitive and/or motor performance. ${ }^{9}$ In contrast, however, some studies have provided evidence to support the notion that information processing stages can occur simultaneously, i.e. in parallel, which means serial processing is not necessarily required. ${ }^{10}$ Furthermore, several afferent and efferent pathways in the central nervous system have parallel pathways, and their distributed parallel processes might clarify certain features of human behavior. ${ }^{11}$

The examination of event-related potentials (ERP) provides useful insights into the nature, organization and timing of neuronal events
Correspondence: Sergio Machado, Institute of Psychiatry, Federal University of Rio de Janeiro, Rua Ferreira Viana, 62/apt, 60. Flamengo, CEP 22210-040, Brazil.

Tel. +55.2191 .567006$

E-mail: secm80@yahoo.com.br

Key words: decision making, event-related potentials, N200, P200, P300.

Contributions: CP, RP, PR designed, conducted the literature review and drafted most of the manuscript. ATS, OAC, MO, MAAL, SM, JGS, MC, ACOS, and AEN performed the literature review and the drafting of the manuscript. All authors were equally involved in reading and approving the final manuscript.

Conflict of interests: the authors declare no conflict of interests.

Received for publication: 13 April 2012.

Revision received: 23 July 2012.

Accepted for publication: 30 July 2012.

This work is licensed under a Creative Commons Attribution NonCommercial 3.0 License (CC BYNC 3.0).

(C) Copyright C. Portella et al., 2012

Licensee PAGEPress, Italy

Neurology International 2012; 4:e16

doi:10.4081/ni.2012.e16

that subserve the sensory, perceptual and cognitive processes. Hence, the different stages of sensory information processing are represented by specific ERP components, specifically reflecting the primary and secondary processing of sensory input, encoding, classification and guidance of the task, as well as the selection and response execution. ${ }^{12}$ ERPs are welldefined representatives of different stages of sensory information processing, with the early ERP components reflecting basic sensory processing of stimuli (lower level processing), e.g. P200 and N200 waves, and the later ERP components reflecting the perceptual and cognitive processing of stimuli (higher level processing), e.g. premotor potential (PMP) and P300 wave that can be transformed into a motor (Go) or into inhibition response (NoGo).13,14 In the current study, we aimed to investigate possible correlations among these early and late stages of information processing by assessing the latency and amplitude of early and late ERP components, including P200, N200, PMP and P300, in the context of a visual oddball paradigm. In addition, we assessed the specific relation of these ERP components to the behavioral measures of our paradigm. We were specifically interested in identifying specific correlations across early and late ERP 
components representing early versus late stages of information processing and, if so, which characteristics (latency, amplitude) demonstrate such relationships in the context of our paradigm.

\section{Materials and Methods}

\section{Subjects}

Twenty healthy subjects [10 male, 10 female; mean age 33.5 years, standard deviation (SD) 11.5] were recruited. All subjects were right handed and had normal or corrected to normal vision. ${ }^{15}$ Inclusion criteria were: absence of mental or physical impairments and no history of psychoactive or psychotropic substance use (screened by an anamnesis and a clinical examination). Furthermore, subjects were not included if they had less than $6-8 \mathrm{~h}$ of sleep prior to the experiment and/or caffeine during the $48 \mathrm{~h}$ prior to the experiment. The entire experimental protocol was explained to all subjects who gave their signed consent before participating in this study. This study was approved by the Ethics Committee at the Federal University of Rio de Janeiro, Brazil.

\section{Stimuli}

In order to minimize sensory interference, the experiment was performed in a sound and light-attenuated room. Participants were seated on a comfortable chair to minimize muscular artifacts while electroencephalography
(EEG) data was collected. During the visual task, lights were turned off and subjects were instructed to concentrate exclusively on the monitor screen. A 15" Samsung monitor was placed $50 \mathrm{~cm}$ in front of the participant. The visual stimulus was presented on the monitor by the ERP acquisition software, developed in DELPHI 5.0. To elicit the P300, all subjects were presented with the same visual discrimination task, which used the classical oddball paradigm. ${ }^{2}$ In this paradigm, two stimuli are presented randomly one of which occurs infrequently. Participants were asked to discriminate targets (25\% infrequent) from non-targets or standard stimuli (75\% frequent). Target stimuli were defined as visual squares and non-targets as circles.

Participants were instructed to respond only to target stimuli by pressing a button with their right index finger using a joystick (Quick Shot-Crystal CS4281, Quick shot, USA). The reaction time (RT) resulting from the pressing of a button in the joystick after each target stimulus was used as an index of motor performance. Participants' reaction times were measured at each trial in milliseconds. Each participant received one block of 350-400 trials. In this block, there was a $95 \%$ chance of 1 4 non-target stimuli preceding a target stimulus and a 5\% chance of 5-7 non-target stimuli preceding a target stimulus. Specifically, 100 target stimuli were presented in the block. The total number of stimuli presented (targets plus non-targets) varied between 350 and 400, and the ratio of target/non target stimuli was 1:4. Each stimulus appeared on the screen for 750 milliseconds with an inter-trial interval (onset to onset) of 1500 milliseconds.

\section{Electroencephalography data acquisition and processing}

The International 10/20 System for electrodes was used with a 20 -channel EEG system (Braintech-3000, EMSA-Medical Instruments, Brazil). 16 The 20 electrodes were arranged in a nylon cap (Electro Cap Inc., Fairfax, VA, USA) yielding monopole derivations referred to linked earlobes. In addition, two $9 \mathrm{~mm}$ diameter electrodes were attached above and on the external corner of the right eye, in a bipolar electrode montage, for eye-movement (EOG) artifact monitoring. Impedance of EEG and E0G electrodes were kept under $5-10 \mathrm{k} \Omega$. The data acquired had total amplitude of less than $100 \mu \mathrm{V}$. The EEG signal was amplified with a gain of 22,000, analogically filtered between $0.01 \mathrm{~Hz}$ (high-pass) and $100 \mathrm{~Hz}$ (low-pass), and sampled at $240 \mathrm{~Hz}$. The software ERP Acquisition (Delphi 5.0), developed at the Brain Mapping and Sensorimotor Integration Laboratory, was employed to filter the raw data: notch $(60 \mathrm{~Hz})$, high-pass of $0.3 \mathrm{~Hz}$ and lowpass of $25 \mathrm{~Hz}$.

To quantify reference-free data, both visual inspection and independent component analysis (ICA) were applied to remove possible sources of artifacts produced by the task. Data from individual electrodes exhibiting loss of contact with the scalp or high impedances $(>10 \mathrm{k} \Omega$ ) were deleted as well as data from single-trial epochs exhibiting excessive movement artifact $( \pm 100 \mu \mathrm{V})$. ICA was then

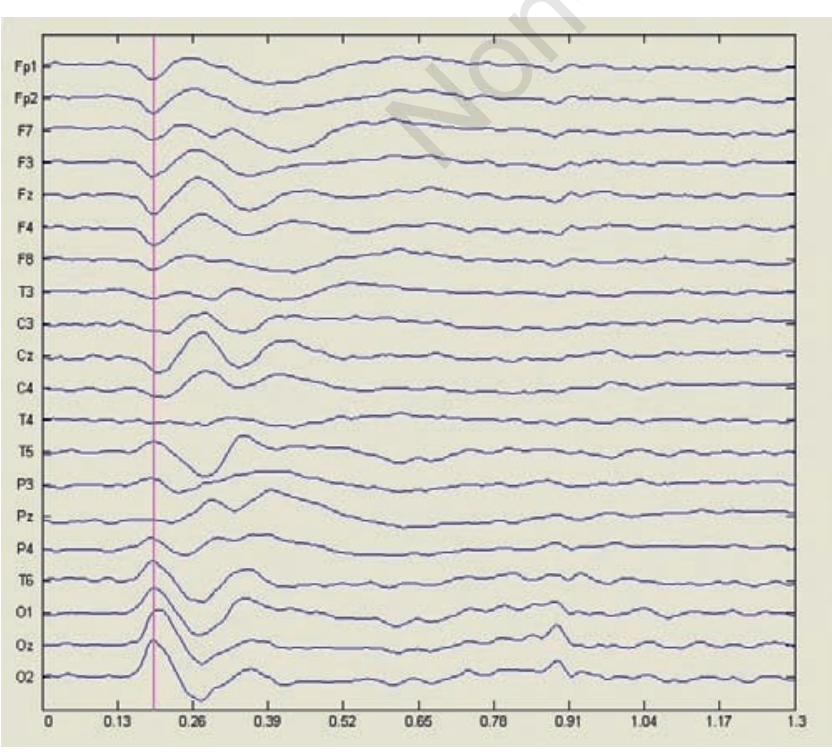

Figure 1. Event-related potential plot for P200 wave.

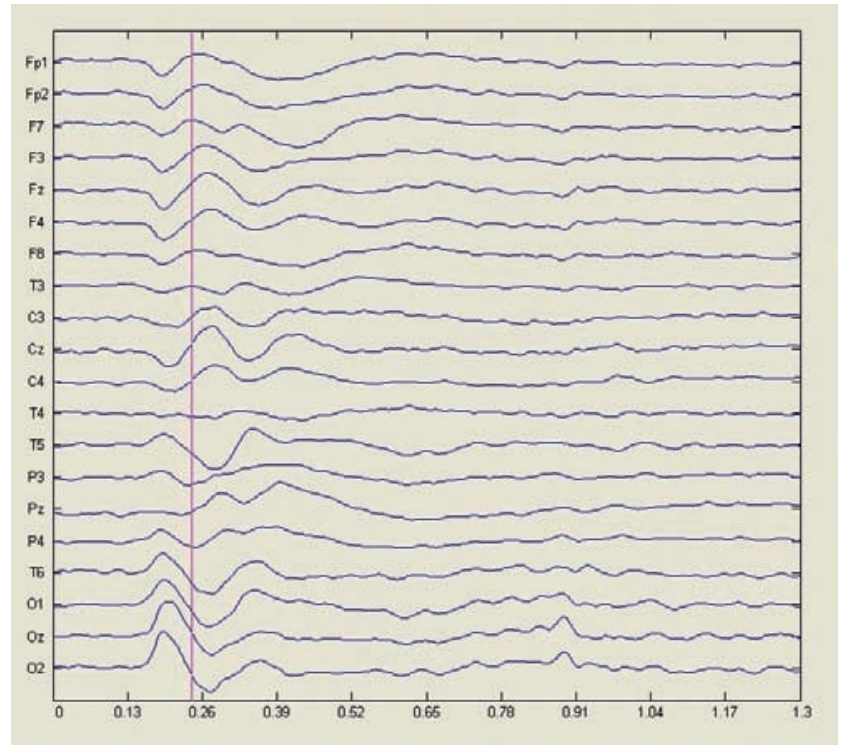

Figure 2. Event-related potential plot for N200 wave. 
applied to identify and remove any remaining artifacts after the initial visual inspection. ICA is an information maximization algorithm that derives spatial filters by blind source separation of the EEG signals into temporally independent and spatially fixed components. Independent components resembling eye-blink or muscle artifact were removed and the remaining components were then back-projected onto the scalp electrodes by multiplying the input data by the inverse matrix of the spatial filter coefficients derived from ICA using established procedures. The ICA-filtered data were then re-inspected for residual artifacts using the same rejection criteria described above. ${ }^{17}$

\section{Statistical analysis}

We obtained current density and RT estimated around the peak latency of each component for each subject. Since the peak latencies varied among subjects, an analyzed time window was determined as the peak latency of each subject \pm SD across subjects. In addition, RT was subsequently averaged to yield a final value for each subject. Missed stimuli were not considered. Although RT is independent of ERP measures, it was used to verify subject alertness during the task. We applied Spearman's correlation analysis $(\mathrm{P} \leq 0.05)$ among latencies and amplitudes of P200 and the other components, e.g. N200, PMP and P300, and between P200 and RT.

\section{Results}

\section{Behavioral data}

During the visual task, all participants responded correctly to the target stimuli (100\%).

\section{Event-related potentials data}

We assessed the specific correlations among the ERP components and the RT of our paradigm. We were interested in identifying specific correlations across early and late ERP components representing early and late stages of information processing and, if such associations were found, determining the characteristics (latency, amplitude) and relationships of those components in the context of our paradigm. Statistical analysis revealed that the latency of the P200 component (mean 200.87 ms; SD 19.7063; Figure 1), showed a significant moderate positive correlation with the latency of N200 (mean $271.56 \mathrm{~ms}$; SD 19.8907; $\mathrm{r}=0.47, \mathrm{P}=0.03$; Figure 2) observed in electrode 02 (Figure $3 \mathrm{~A}$ ); with the latency of PMP (mean $342.62 \mathrm{~ms}$; SD 27.3317; $\mathrm{r}=0.43, \mathrm{P}=0.05$; Figure 4) observed in electrode C3 (Figure 3B); with the latency of the P300 (mean 408.75
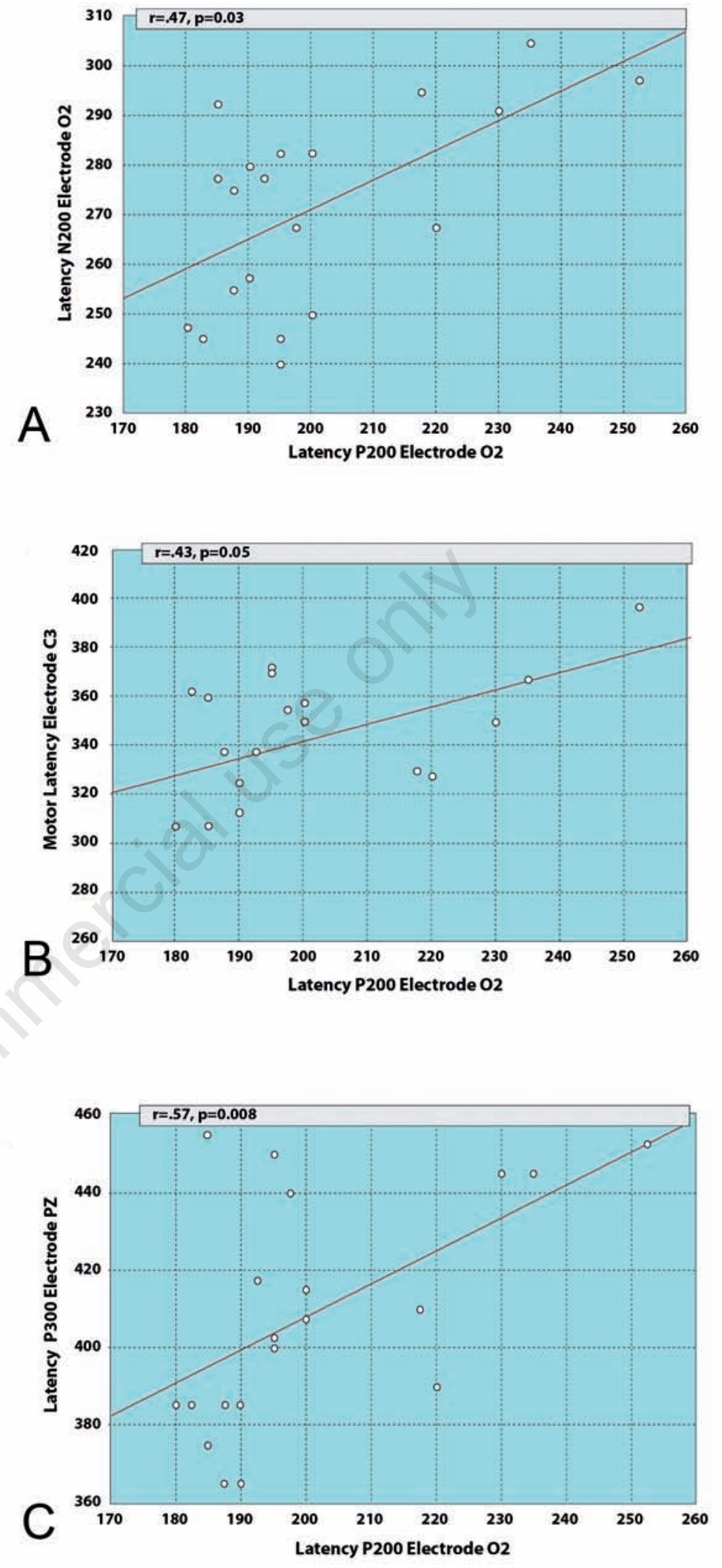

Figure 3. Positive correlation among the latency of P200 (electrode O2), N200 (electrode O2), PMP (electrode C3), P300 (electrode PZ), and the TR. Each data point represents a single unique subject. A) Positive correlation between latency of P200 and N200 waves of the electrode 02 . Significant difference; $r=0.47, P=0.03$; B) positive correlation between latency of P200 wave of the electrode $\mathrm{O} 2$ and motor latency wave of the electrode $\mathrm{C} 3$. Significant difference; $\mathbf{r}=\mathbf{0 . 4 3}, \mathbf{P}=\mathbf{0 . 0 5} ; \mathrm{C}$ ) positive correlation between latency of $\mathbf{P 2 0 0}$ wave of the electrode $\mathrm{O} 2$ and latency $\mathrm{P} 300$ wave of the electrode Pz. Significant difference; $\mathbf{r}=0.57, \mathrm{P}=\mathbf{0 . 0 0 8}$. 
ms; SD 30.0602; r=0.57, $\mathrm{P}=0.008$; Figure 5) observed in electrode $\mathrm{Pz}$ (Figure $3 \mathrm{C}$ ).

In contrast, the amplitude of the P200 component (mean $10.05 \mu \mathrm{V}$; SD 5.3082359; Figure 1), showed a significant moderate negative correlation with the latency of N200 (mean $271.56 \mathrm{~ms}$; SD 19.8907; r=-0.46, $\mathrm{P}=0.04$; Figure 2) observed in electrode 02 (Figure $6 \mathrm{~A}$ ); with the latency of PMP (mean $342.62 \mathrm{~ms}$; SD 27.3317; $r=-0.47, P=0.03$; Figure 4) observed in electrode C3 (Figure 6B), and with the latency of P300 (mean 408.75 ms; SD 30.0602; r=$0.45, \mathrm{P}=0.04$; Figure 5) observed in electrode $\mathrm{Pz}$ (Figure 6C).

\section{Discussion}

The results demonstrate moderate positive correlations among the latency of P200 (electrode 02), N200 (electrode 02), PMP (electrode C3), P300 (electrode PZ). In addition, moderate negative correlation between the amplitude of P200 and the latencies of N200 (electrode 02), PPM (electrode C3), P300 (electrode PZ) was found. With this in mind, the discussion is divided into two parts: first positive correlation and then negative correlation.

\section{Positive correlation}

The P200 component is well-documented as a deflection with maximum positive amplitude between 150 and $250 \mathrm{~ms}$ and is strongly associated with the secondary processing of visual input. ${ }^{18,19}$ On the other hand, the N200 compo- nent is the maximum negative amplitude between 175 and $250 \mathrm{~ms}^{18}$ related to multiple neuronal processes associated with discrimination and classification of visual stimuli., 4,20 The dynamics of these components can be understood from visual routes. According to Kropotov, 21 the visual pathways are composed of hierarchical structures in which signals are transferred from the first level to the others. That is, visual input reaches primary visual cortex (Brodmann area 17) through the lateral geniculate body of thalamus. Then, ventral (temporal) and dorsal (parietal) visual pathways are activated that discriminate different aspects of visual information (Figure 7). The dorsal pathway interests us because it is situated under areas covered by the electrodes $02, \mathrm{PZ}$ and $\mathrm{C} 3$; these include secondary sensory cortices for visual stimuli (Brodmann areas 18, 19, 7 and 5) and primary sensory cortex (Brodmann area 3 ). These areas should be activated sequentially in order to process information related to the speed and position of visual information. Therefore, according to the hierarchic theory of visual pathways, it is possible to infer that the earlier secondary processing of visual input occurs (register of input - P200 latency), the faster discrimination and the classification process of input will happen (N200 latency).

The PMP has negative amplitude that appears approximately $300 \mathrm{~ms}$ before the start of the movement with a peak occurring at 100 ms. ${ }^{22}$ It is clearly observed in the contralateral motor area relative to movement and has been utilized to indicate the stage of motor execution processing of ERP.23-25
According to a recent study, the parietal cortex would be involved not only in the spatial demands of the visual scene but also in the visual organization for motor procedures. ${ }^{21}$ Posterior parietal sites are interconnected with the pre-motor cortex in order to prepare the individual to start eye movement, to aim the eyes towards the selected object, and then to reach and manipulate it. Within this context, the dorsal pathway (parietal) would be related to the procedural memory. Therefore, it is suggested that as earlier registration of visual input (P200 latency) occurs, the motor answer processing happens faster (PPM latency).

P300 has positive maximum amplitude of between 250 and $600 \mathrm{~ms}$ and rises after the conclusion of a task related to stimuli differentiation reflecting attention reorganization. 18,26 It is also related to working memory updating and learning processes. ${ }^{27}$ It has been suggested that this potential is elicited at the end of a cognitive episode in which the decision is made as to whether the stimulus is important or not. 28 P300 latency may reflect the discrimination time needed to interpret a stimulus as important or unimportant. ${ }^{29}$

Another study evaluated working memory (WM) in patients with Amyotrophic Lateral Sclerosis through ERPs in the context of an auditory oddball paradigm. ${ }^{30}$ The authors found a prolonged latency of the N200 and P300 components in $60 \%$ of subjects and proposed that P300 delayed latency indicates a WM impairment. Those results corroborate the visual pathways theory. ${ }^{21}$ First, they agree that the dorsal visual pathway is involved with the

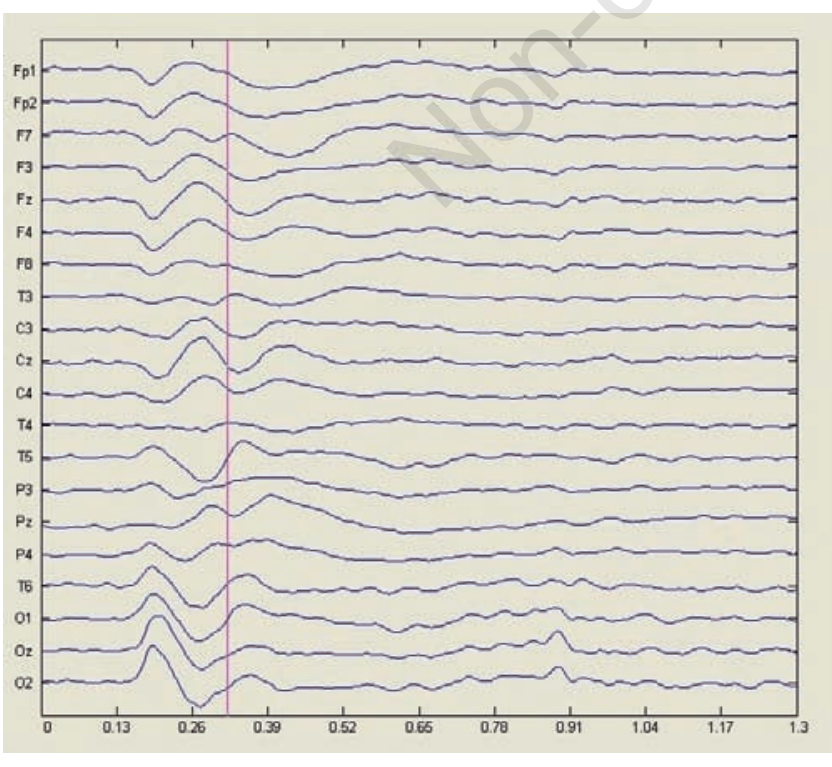

Figure 4. Event-related potential plot for premotor potential wave.

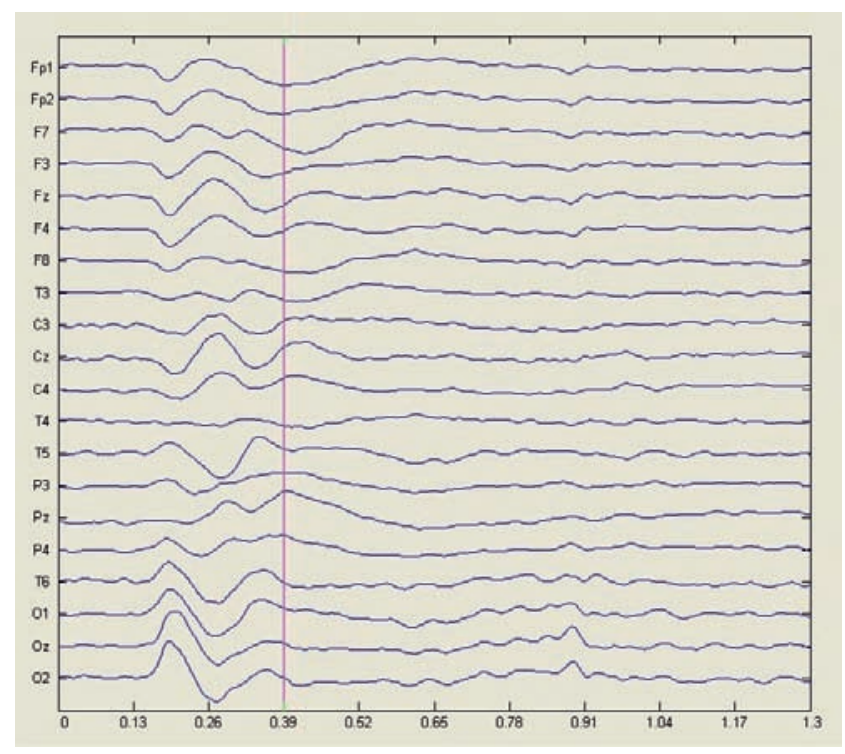

Figure 5. Event-related potential plot for P300 wave. 

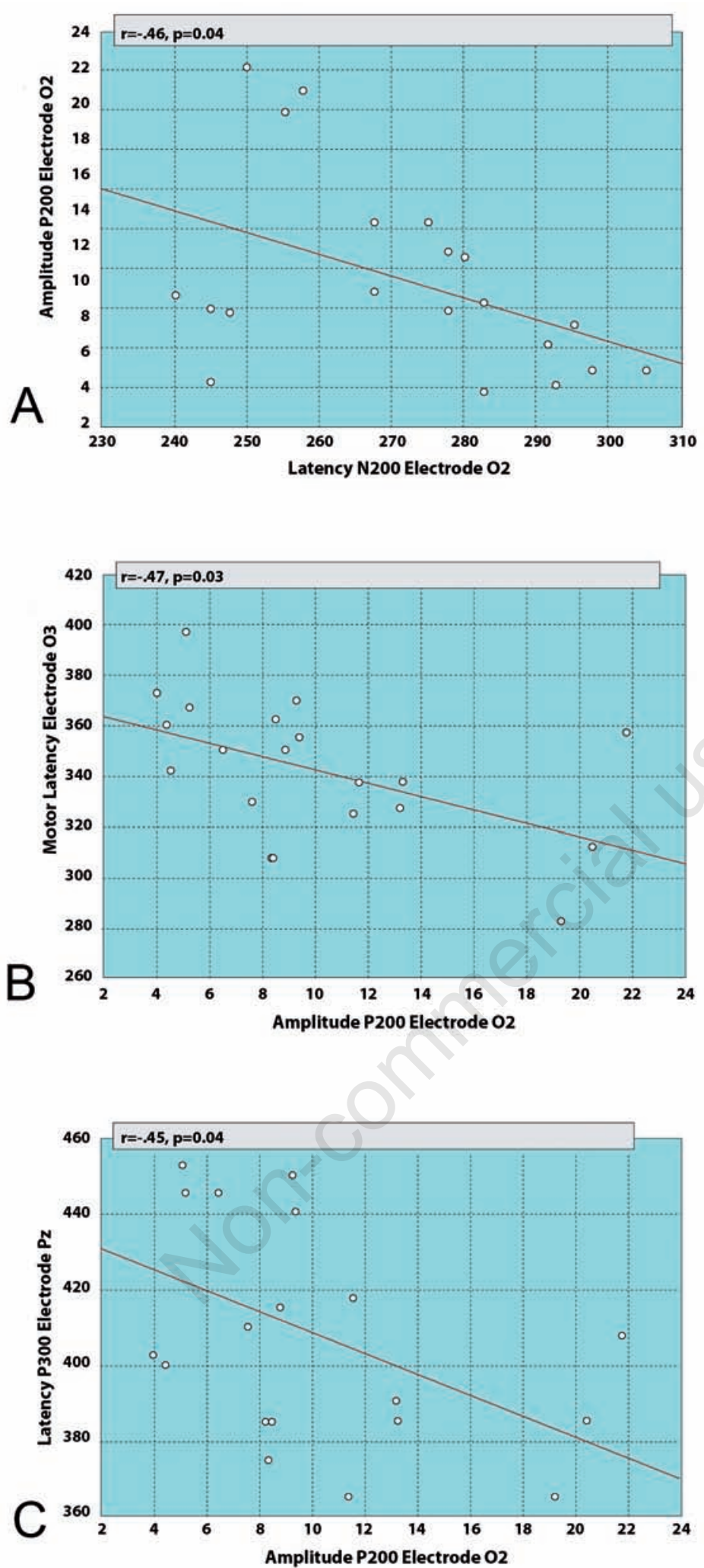

Figure 6. Negative correlation between the amplitude of P200 and the latencies of N200 (electrode O2), premotor potential (electrode C3), P300 (electrode PZ). Each data point represents a single unique subject. A) Negative correlation between amplitude of P200 and latency of $\mathrm{N} 200$ waves of the electrode $\mathrm{O} 2$. Significant difference; $r=-0.46, P=0.04 ; B$ ) negative correlation between motor latency wave of the electrode $\mathrm{C} 3$ and amplitude of P200 wave of the electrode $\mathrm{O} 2$. Significant difference; $\mathrm{r}=-\mathbf{0 . 4 7}, \mathrm{P}=\mathbf{0 . 0 3} \mathrm{C}$ ) negative correlation between latency of the $\mathrm{P} 300$ wave of the electrode $\mathrm{Pz}$ and amplitude of $\mathrm{P} 200$ wave of the electrode $\mathrm{O} 2$. Significant difference; $\mathrm{r}=-\mathbf{0 . 4 5}, \mathrm{P}=\mathbf{0 . 0 4}$.
WM. Second, they reinforce the hierarchic theory that makes up these pathways. In other words, signals are transferred from the first level to the other levels, in only this order. In this way, it is suggested that the earlier visual input register occurs (P200 latency), the faster attention reorganization and the working memory update takes place (P300 latency).

Anjana and colleagues assessed cognitive status in children with attention deficit hyperactivity disorder (ADHD) using auditory ERPs. ${ }^{31}$ The ADHD children showed a statistically significant N200 latency prolongation and amplitude decrease when compared with controls. N100, P200 and P300 were prolonged in ADHD children, but the difference versus controls was without statistical significance. The authors suggested dysfunctions in the discrimination of task-relevant stimuli could explain their findings. These findings support the idea that as attention level is lower, stimuli discrimination capacity is also reduced. Conversely, as attention level is higher, activation level of occipital areas involved in the secondary processing of the visual input (P200 amplitude) is also enhanced, and consequently ERP components, including N200 (discrimination and classification process of visual input) have shorter latency.

The results of Anjana et al. ${ }^{31}$ showed a significantly longer RT in ADHD subjects as compared with controls. Furthermore, using the same idea as above, attention deficits could lead to a lower cortical activation level for the input register (lower P200 amplitude) and consequently to an RT delay. In this sense, in normal attention levels, a negative correlation between P200 amplitude and RT would be expected. In another way, the higher the activation level of occipital areas involved in the secondary processing of visual input (P200 amplitude), the lower would be the RT. Looking to the fact that RT is preceded by PMP (motor response processing), a negative correlation between P200 amplitude and PMP latency would also be expected. In other words, as attention level is higher, and consequently the activation of cortical areas involved in visual input register (P200 amplitude), PMP latency is shorter.

Vandoolaeghe and colleagues used auditory event related potentials (AERPs) to study patients with major depression without cognitive deterioration and patients with major depression with Alzheimer's dementia and cognitive impairment. ${ }^{29}$ It was observed that clinically depressed subjects without cognitive deterioration had significantly higher P200 amplitude and P300 latency than normal volunteers. Patients with Alzheimer's dementia and depression with cognitive impairment had a significantly higher P300 latency than depressed patients without cognitive impairment. Apart from the differences in methodology (auditory 

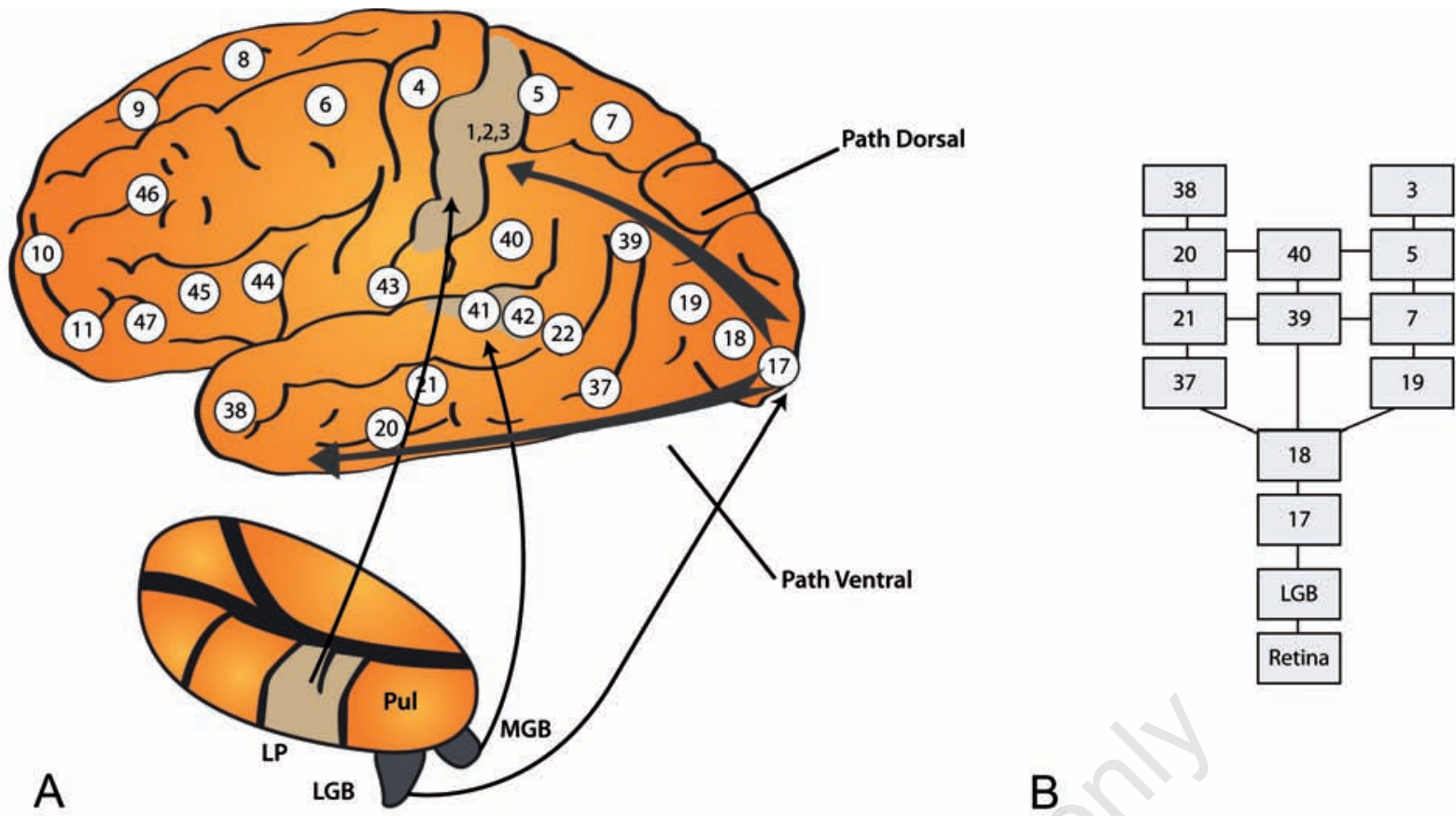

Figure 7. Cortical projections of dorsal and ventral streams in the visual modality in the cerebral cortex. A) Sensory-related thalamic nuclei (bottom) serve as gates to the primary sensory areas (top) in thecortex. B) Schematic representation of ventral and dorsal streams. LGB, lateral geniculate body, MGB, medial geniculate body, LP, lateral posterior nucleus.

vs visual oddball, patients $v s$ normal volunteers), the results of Vandoolaeghe and colleagues conflict with our data. While there seems to be a positive correlation between P200 amplitude and P300 latency, the present study found a negative correlation. Based on our findings, it is suggested that as activation level of occipital areas involved in the secondary processing of visual input (P200 amplitude) is higher, attention reorganization and working memory update (P300 latency) is faster.

\section{Conclusions}

Our results contribute to a better understanding of the relations between early and late stages of information processing. We speculated that if the secondary processing of visual input (P200 latency) occurs faster, the following will also happen sooner: discrimination and classification process of this input (N200 latency), motor response processing (PMP latency), reorganization of attention and working memory update (P300 latency), and RT. N200, PMP and P300 latencies are also anticipated when higher activation level of occipital areas involved in the secondary processing of visual input rise (P200 amplitude). We also propose that P200 amplitude and latency influenced the latencies, but not amplitudes, of N200, PMP and
P300. Furthermore, we also suggest that RT is influenced by latency, not amplitude, of P200. New investigations involving the correlation between ERP components, including P200, N200, PMP and P300 in the context of a visual oddball paradigm with healthy subjects are required to test these hypotheses.

\section{References}

1. Polich J, Criado JR. Neuropsychology and neuropharmacology of P3a and P3b. Int J Psychophysiol 2006;60:172-85.

2. Polich J. Updating P300: an integrative theory of P3a and P3b. Clin Neurophysiol 2007;118:2128-48.

3. Rossini PM, Rossi S, Babiloni C, Polich J. Clinical neurophysiology of aging brain: from normal aging to neurodegeneration. Prog Neurobiol 2007;83:375-400.

4. Olofsson JK, Nordin S, Sequeira H, Polich J. Affective picture processing: an integrative review of ERP findings. Biol Psychol 2008;77:247-65.

5. Rozenkrants B, Polich J. Affective ERP processing in a visual oddball task: arousal, valence, and gender. Clin Neurophysiol 2008;119:2260-5.

6. Pontifex MB, Hillman CH, Polich J. Age, physical fitness, and attention: P3a and P3b. Psychophysiology 2009;46:379-87.
7. Kaestner EJ, Polich J. Affective recognition memory processing and event-related brain potentials. Cogn Affect Behav Neurosci 2011;11:186-98.

8. Basar-Eroglu C, Demiralp T, Schurmann M, Basar E. Topological distribution of oddball 'P300' responses. Int J Psychophysiol 2001;39:213-20.

9. Di Russo F, Bultrini A, Brunelli S, et al. Benefits of sports participation for executive function in disabled athletes. $\mathrm{J}$ Neurotrauma 2010;27:2309-19.

10. Duncan CC, Barry RJ, Connolly JF, et al. Event-related potentials in clinical research: guidelines for eliciting, recording, and quantifying mismatch negativity, P300, and N400. Clin Neurophysiol 2009; 120:1883-908.

11. Sadaghiani S, Hesselmann G, Friston KJ, Kleinschmidt A. The relation of ongoing brain activity, evoked neural responses, and cognition. Front Syst Neurosci 2010; 4:20.

12. Wang L, Kuroiwa Y, Li M, et al. Do P1 and N1 evoked by the ERP task reflect primary visual processing in Parkinson's disease? Doc Ophthalmol 2001;102:83-93.

13. Banaschewski T, Brandeis D. Annotation: what electrical brain activity tells us about brain function that other techniques cannot tell us - a child psychiatric perspective. J Child Psychol Psychiatry 2007;48:415-35.

14. Kotchoubey B, Lang S. Event-related poten- 
tials in an auditory semantic oddball task in humans. Neurosci Lett 2001;310: 93-6.

15. Oldfield RC. The assessment and analysis of handedness: the Edinburgh inventory. Neuropsychologia 1971;9:97-113.

16. Klem GH, Luders HO, Jasper HH, Elger C. The ten-twenty electrode system of the International Federation. The International Federation of Clinical Neurophysiology. Electroencephalogr Clin Neurophysiol Suppl 1999;52:3-6.

17. Delorme A, Makeig S. EEGLAB: an open source toolbox for analysis of single-trial EEG dynamics including independent component analysis. J Neurosci Methods 2004;134:9-21.

18. Golob EJ, Starr A. Effects of stimulus sequence on event-related potentials and reaction time during target detection in Alzheimer's disease. Clin Neurophysiol 2000;111:1438-49.

19. Frodl T, Meisenzahl EM, Gallinat J, et al. Markers from event-related potential subcomponents and reaction time for information processing dysfunction in schizophrenia. Eur Arch Psychiatry Clin Neurosci 1998;248:307-13.

20. Heinze HJ, Munte TF, Gobiet W, et al.
Parallel and serial visual search after closed head injury: electrophysiological evidence for perceptual dysfunctions. Neuropsychologia 1992;30:495-514.

21. Kropotov JD, Ponomarev VA. Decomposing N2 NOGO wave of event-related potentials into independent components. Neuroreport 2009;20:1592-6.

22. Ulrich R, Rinkenauer G, Miller J. Effects of stimulus duration and intensity on simple reaction time and response force. J Exp Psychol Hum Percept Perform 1998;24: 915-28.

23. Libet B, Wright EW, Gleason CA. Readiness-potentials preceding unrestricted 'spontaneous' vs. pre-planned voluntary acts. Electroencephalogr Clin Neurophysiol 1982;54:322-35.

24. Hsieh $\mathrm{S}$. The lateralized readiness potential and P300 of stimulus-set switching. Int J Psychophysiol 2006;60:284-91.

25. Touzalin-Chretien P, Ehrler S, Dufour A. Dominance of vision over proprioception on motor programming: evidence from ERP. Cereb Cortex 2010;20:2007-16.

26. Felmingham KL, Bryant RA, Kendall C, Gordon E. Event-related potential dysfunction in posttraumatic stress disorder: the role of numbing. Psychiatry Res 2002;109: 171-9.

27. Martin FH, Garfield J. Combined effects of alcohol and caffeine on the late components of the event-related potential and on reaction time. Biol Psychol 2006;71:63-73.

28. Desmedt JE, Debecker J. Slow potential shifts and decision P350 interactions in tasks with random sequences of nearthreshold clicks and finger stimuli delivered at regular intervals. Electroencephalogr Clin Neurophysiol 1979;47: 671-9.

29. Vandoolaeghe E, van Hunsel F, Nuyten D, Maes M. Auditory event related potentials in major depression: prolonged P300 latency and increased P200 amplitude. J Affect Disord 1998;48:105-13.

30. Grill-Spector K, Henson R, Martin A. Repetition and the brain: neural models of stimulus-specific effects. Trends Cogn Sci 2006;10:14-23.

31. Anjana Y, Khaliq F, Vaney N. Event-related potentials study in attention deficit hyperactivity disorder. Funct Neurol 2010;25:8792. 